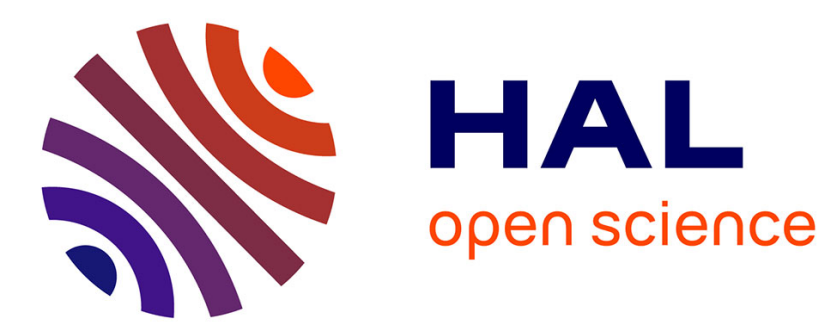

\title{
Linear and nonlinear waveguiding of few-cycle optical solitons in a planar geometry
}

Hervé Leblond, Dumitru Mihalache

\section{To cite this version:}

Hervé Leblond, Dumitru Mihalache. Linear and nonlinear waveguiding of few-cycle optical solitons in a planar geometry. Physical Review A : Atomic, molecular, and optical physics [1990-2015], 2013, 88 (2), pp.023840. 10.1103/PhysRevA.88.023840 . hal-03204244

\section{HAL Id: hal-03204244 \\ https://univ-angers.hal.science/hal-03204244}

Submitted on 21 Apr 2021

HAL is a multi-disciplinary open access archive for the deposit and dissemination of scientific research documents, whether they are published or not. The documents may come from teaching and research institutions in France or abroad, or from public or private research centers.
L'archive ouverte pluridisciplinaire HAL, est destinée au dépôt et à la diffusion de documents scientifiques de niveau recherche, publiés ou non, émanant des établissements d'enseignement et de recherche français ou étrangers, des laboratoires publics ou privés. 


\title{
Linear and nonlinear waveguiding of few-cycle optical solitons in a planar geometry
}

\author{
Hervé Leblond ${ }^{1}$ and Dumitru Mihalache $e^{2,3}$ \\ ${ }^{1}$ LUNAM Université, Université d'Angers, Laboratoire de Photonique d'Angers, EA 4464, 2 Boulevard Lavoisier, 49000 Angers, France \\ ${ }^{2}$ Academy of Romanian Scientists, 54 Splaiul Independentei, RO-050094 Bucharest, Romania \\ ${ }^{3}$ Horia Hulubei National Institute for Physics and Nuclear Engineering, 30 Reactorului, RO-077125 Magurele-Bucharest, Romania
}

(Received 6 May 2013; published 22 August 2013)

\begin{abstract}
We consider the guiding of a few-cycle optical soliton by total internal reflexion, in a planar geometry. By means of numerical solution of a cubic generalized Kadomtsev-Petviashvili equation, we show that, for intensities high enough to induce soliton formation, the nonlinear effects considerably widen the guided mode and can even prevent guiding for the shortest pulses and the narrowest waveguides. However, waveguiding can be achieved by means of a steep variation of the nonlinear coefficients, e.g., by using a higher nonlinear coefficient in the cladding than that in the waveguide core. We further propose an analytical approach for extremely narrow guides, which allows us to derive a modified Korteweg-de Vries-type model for the propagation of few-cycle optical solitons in the planar waveguide.
\end{abstract}

DOI: 10.1103/PhysRevA.88.023840

PACS number(s): 42.65.Tg, 42.65.Sf, 47.20.Ky

\section{INTRODUCTION}

Self-trapped spatiotemporal wave packets, which form in both conservative and dissipative nonlinear optical media, have been extensively investigated during the past decade. Such localized optical waves, alias optical solitons, occur in diverse physical settings, and numerous studies of their existence, generation, and robustness to perturbations have been reported in the fast growing area of nonlinear optics and photonics [1-5]. Owing to the continuous advances in both the generation and control of ultrashort optical pulses from Kerr-lens mode-locked Ti:sapphire lasers, with duration of only a few optical cycles, the study of propagation of such intense few-cycle pulses (FCPs) has become a matter of growing research interest over the past several years. The experimental progress in the area of FCPs has paved the way for the development of new theoretical approaches to model their formation and to study their robustness to propagation in a series of physical settings. It is worth noting that in the case of wave packets containing only a few optical cycles, the adequate analysis of the spatiotemporal pulse dynamics should be carried out beyond the conventional slowly varying envelope approximation (SVEA); see a series of earlier works in this area [6-12] and a recent overview of theoretical models of few-cycle solitons beyond the SVEA [13].

Since the standard SVEA is not valid for describing the spatiotemporal evolution of ultrashort pulses containing only a few oscillations of the optical field, several research groups derived alternative approaches for describing the physics of such phenomena; see a list of recent relevant works in this growing area [14-28]. However, though most of the past research interest in the study of propagation of FCPs was confined to the simplest two-level atomic system (see the review [13]), a more realistic description of the ultrashortsoliton propagation in optical media by taking into account an arbitrary number of atomic levels was also put forward [29-31]. Thus, in a series of recent papers [29-31], some of the main results concerning the systematic use of the reductive expansion method beyond the SVEA in the simplest case of two-level atoms were extended to few-cycle optical solitons in media described by a generic atomic Hamiltonian, i.e., it was considered a general quantum model involving an arbitrary number of energy levels.

Though a variety of optical solitons that involve either temporal effects (e.g., solitons in monomode fibers) or spatial effects (e.g, solitons in photorefractive media) have been observed during the past three decades [1-5], the experimental studies of localized optical structures that involve both degrees of freedom, the so-called spatiotemporal optical solitons (alias nonlinear light bullets), are quite rare [32-38]. However, there is a continuous flow of theoretical works in the area of linear and nonlinear light bullets; see the early seminal works [39-43] and a few recent review papers $[5,44,45]$. Such spatiotemporal optical solitons attract a lot of interest owing to their potential for all-optical logic devices with switching rates of several $10^{12} \mathrm{~Hz}$, i.e., with terahertz switching speeds. We also mention theoretical studies of ultrashort spatiotemporal optical pulse propagation in cubic (Kerr-like) media without the use of SVEA [46,47], and of ultrashort spatiotemporal optical solitons in quadratic nonlinear media $[48,49]$. Also, the formation of robust ultrashort (only two cycles long) spatiotemporal optical solitons in carbon nanotube arrays has also been considered in a recent work [50].

Although many studies are devoted to short pulse propagation in waveguides, almost none of them consider the process of waveguiding itself: usually the waveguide is treated using linear modes and effective refractive index (see, e.g., [51]), which is valid in principle in SVEA only, and assuming that the index variation due to the nonlinear effect is small with respect to the index difference responsible for waveguiding (or the equivalent effect in photonic crystals or fibers). Even within the SVEA, only a few studies considering the guiding process itself in a nonlinear way, e.g., in inhomogeneous nonlinear media in the form of graded-index materials with cubic (Kerr) nonlinearities have been reported in the past $[52,53]$. In this research line, a recent work [38] reports the observation of spatiotemporal optical solitons with $0.5 \mathrm{~nJ}$ pulse energy in a cylindrical waveguiding geometry (a graded-index multimode optical fiber).

We consider here the waveguiding process of an ultrashort pulse beyond the SVEA in the most simple configuration, which is the step-index planar waveguide. We perform 
numerical and analytical modeling of the propagation of ultrashort solitons described by the cubic generalized KadomtsevPetviashvili (CGKP) equation in a planar waveguide geometry based on total internal reflection. We consider the situation when the nonlinear cubic $\left(\chi^{(3)}\right)$ medium is a self-defocusing one in the transverse spatial dimension while temporal selfcompression of the pulse occurs. Assuming a large waveguide core, the evolution of the electric field in the nonlinear medium is described by a CGKP equation, which contains an additional term involving the cladding velocity shift. We show that nonlinear wave propagation of ultrashort (few-cycle long) spatiotemporal wave packets in planar waveguides is possible when the cladding exhibits a sufficiently high nonlinear refractive index. We also give an analytic theory of the guided few-cycle solitons in ultranarrow planar nonlinear waveguides by using the powerful reductive perturbation method.

This paper is organized as follows. In the next section, we introduce the CGKP model for ultrashort-soliton propagation in a planar waveguide geometry and we obtain the corresponding linear guided modes and the associated waveguiding condition. In Sec. III, we numerically solve the CGKP equation by considering adequate input data in the form of a fundamental guided mode multiplied by a temporal Gaussian pulse. Computation of the confinement factor allows us to make prominent the nonlinear widening of the guided mode. Then, in Sec. IV, we consider in detail the nonlinear waveguiding process as a function of the difference between the two nonlinear coefficients in the waveguide core and the cladding. We also compare the confinement factor in the nonlinear waveguide with that corresponding to the linear guide. In Sec. V, we present some analytic calculations of the guided few-cycle solitons in the case of ultranarrow planar waveguides. Finally, in Sec. VI, we present our conclusions.

\section{BASIC EQUATIONS}

We consider a planar optical waveguide. The spatial coordinate frame is denoted by $(O \xi \eta \zeta)$, the propagation direction is the $\zeta$ axis, the waveguide core is limited by the planes $\xi= \pm a$, and the system is translationally invariant along $\eta$. We consider a planar geometry for the sake of simplicity and of computation time; however, the extension to other relevant situations of both rectangular and cylindrical channel waveguides is straightforward.

The evolution of the electric field $E$ in the medium is described by the cubic generalized Kadomtsev-Petviashvili (CGKP) equation [29,46,54],

$\partial_{\zeta} E+\beta_{\alpha} \partial_{\tau}^{3} E+\gamma_{\alpha} \partial_{\tau} E^{3}+\frac{1}{V_{\alpha}} \partial_{\tau} E-\frac{V_{\alpha}}{2} \int^{\tau} \partial_{\xi}^{2} E d \tau^{\prime}=0$,

with $\alpha=1$ in the waveguide core $(-a \leqslant x \leqslant a)$ and $\alpha=2$ in the cladding $(|x| \geqslant a)$. The nonlinear coefficient is

$$
\gamma_{\alpha}=\frac{1}{2 n_{\alpha} c} \chi_{\alpha}^{(3)}
$$

where $n_{\alpha}(\alpha=1,2)$ are the linear refractive indices, $\chi_{\alpha}^{(3)}(\alpha=$ $1,2)$ are the third-order susceptibilities at the low-frequency limit, and the dispersion parameters are

$$
\beta_{\alpha}=\frac{\left(-n_{\alpha}^{\prime \prime}\right)}{2 c},
$$

where the prime denotes the derivative with respect to $\omega$.

It is more convenient, for the numerical study of the equations, to consider a dimensionless form of the CGKP equation (1):

$$
\partial_{z} u=A_{\alpha} \partial_{t}^{3} u+B_{\alpha} \partial_{t} u^{3}+v_{\alpha} \partial_{t} u+\frac{W_{\alpha}}{2} \int^{t} \partial_{x}^{2} u d t^{\prime},
$$

in which the dimensionless variables are defined as $z=\zeta / L$, $t=\left(\tau-\zeta / V_{0}\right) / \tau_{w}, x=\xi / l$, and $u=E / E_{0}$. The quantities $L$ and $l$ are longitudinal and transversal reference lengths, and $\tau_{w}$ and $E_{0}$ are reference time and reference electric field, respectively. The velocity $V_{0}=c / n_{0}$ is chosen to be close to both velocities $V_{1}$ and $V_{2}$, and these latter velocities are replaced with $V_{0}$ in front of the antiderivative (integral) term in Eq. (1). The coefficients then read

$$
\begin{gathered}
A_{\alpha}=\frac{\left(-\beta_{\alpha} L\right)}{\tau_{w}^{3}}, \\
B_{\alpha}=\frac{\left(-\gamma_{\alpha} L E_{0}^{2}\right)}{\tau_{w}}, \\
v_{\alpha}=\frac{L}{\tau_{w}}\left(\frac{1}{V_{0}}-\frac{1}{V_{\alpha}}\right),
\end{gathered}
$$

and

$$
W_{\alpha}=\frac{V_{\alpha} L \tau_{w}}{l^{2}} \simeq \frac{V_{0} L \tau_{w}}{l^{2}} .
$$

The reference time $\tau_{w}$ is fixed arbitrary of the order of magnitude of the optical period and pulse length, i.e., we work in the femtosecond range. Then we can set $A_{\alpha}=1$ by the choice of the longitudinal reference length,

$$
L=\frac{\tau_{w}^{3}}{\left(-\beta_{\alpha}\right)}=\frac{2 c \tau_{w}^{3}}{n_{\alpha}^{\prime \prime}}
$$

Obviously, we can set $A_{1}=A_{2}$ only if the dispersion coefficients of both media are the same, i.e., $n_{1}^{\prime \prime}=n_{2}^{\prime \prime}$. The nonlinear coefficient $B_{\alpha}$ can then be set to 1 by choosing the reference electric field as

$$
E_{0}=\sqrt{\frac{\tau_{w}}{(-\gamma L)}} .
$$

If $L$ has been fixed according to Eq. (9), then Eq. (10) becomes

$$
E_{0}=\sqrt{\frac{n_{\alpha} n_{\alpha}^{\prime \prime}}{\left(-\chi_{\alpha}^{(3)} \tau_{w}^{2}\right)}} .
$$

Equation (11) requires $n_{\alpha}^{\prime \prime} \chi_{\alpha}^{(3)}<0$, which is well known as the condition for temporal pulse compression to occur.

In fact, we need to restrict ourselves to the situation in which the nonlinear effect is a spatially self-defocusing one while temporal compression occurs. The latter condition is necessary for temporal soliton formation, which is the situation we intend to consider in this paper. It is worthwhile to mention that if spatial self-focusing occurs, it leads to the collapse of ultrashort 
spatiotemporal pulses [46]. Numerical computation allows one to check that collapse will occur inside the waveguide, which we intend to avoid. This situation differs from the usual formation of temporal solitons in optical fibers, as described within the SVEA; let us say, to make it more concrete, that we consider picosecond temporal solitons in a standard telecom fiber. For picosecond fiber solitons, the longitudinal size of the pulse is very large with respect to the wavelength (10 ps correspond to $3 \mathrm{~mm}$ ), while its transverse size has the same order of magnitude as the wavelength (the core diameter of the standard telecom fiber is about $8.2 \mu \mathrm{m}$ ). Hence the picosecond pulse propagation is a one-dimensional problem. The pulse width, as confined by the optical fiber, is already narrower than that of a pulse of the same length which would collapse in a bulk medium. For a FCP, the situation strongly differs since the pulse length has the same order of magnitude as the wavelength. Hence the pulse propagation in the waveguide is, in fact, a tridimensional problem (in our model, it is a bidimensional one), and, consequently, collapse can occur in the same way as in bulk media. Hence we need to restrict to negative (self-defocusing) nonlinearity $\left(\chi_{\alpha}^{(3)}<0\right)$ and to normal group-velocity dispersion $\left(n_{\alpha}^{\prime \prime}>0\right)$, which ensure that $L$ given by Eq. (9) is positive and $E_{0}$ given by Eq. (10) is a real number. According to (6) and (2),

$$
\frac{B_{2}}{B_{1}}=\frac{\gamma_{2}}{\gamma_{1}}=\frac{n_{1} \chi_{2}^{(3)}}{n_{2} \chi_{1}^{(3)}} \text {. }
$$

If we fix $E_{0}$ according to Eq. (10) for $\alpha=1$, so that $B_{1}=1$, then Eq. (12) gives $B_{2}$. We assume some variation $\delta n_{\alpha}$ of the optical refractive index, so that $n_{\alpha}=n_{0}+\delta n_{\alpha}$, with $\delta n_{\alpha} \ll 1$. Then the relative velocity is proportional to $\left(-\delta n_{\alpha}\right)$, as

$$
v_{\alpha}=\frac{\left(-\delta n_{\alpha} L\right)}{t_{w} c} .
$$

If $L$ is defined according to (9), then Eq. (13) becomes

$$
v_{\alpha}=\frac{2 t_{w}^{2} \delta n_{\alpha}}{n_{\alpha}^{\prime \prime}}
$$

Linear guided modes are easily computed: we seek solutions of Eq. (4) of the form $u=f(x) \exp \left[i\left(\omega t-k_{z} z\right)\right]$. It is found that $f$ must satisfy the equation $\partial_{x}^{2} f+K_{\alpha} f=0$, with

$$
K_{\alpha}=\frac{2 \omega}{V_{\alpha}}\left(A_{\alpha} \omega^{3}-k_{z}-\omega v_{\alpha}\right) .
$$

Due to the symmetry of the setup, the solutions must have some parity; we restrict ourselves to even volume modes, of the form $f=R \cos \left(k_{x} x\right)$ with $k_{x}^{2}=K_{1}$ in the core, and $f=C e^{-\kappa|x|}$ with $\kappa^{2}=-K_{2}^{2}$ in the cladding. Continuity conditions of electromagnetic fields at the boundaries between the waveguide core and cladding imply that both $f(x)$ and $\partial_{x} f(x)$ are continuous at $x= \pm a$, and we get the waveguide dispersion relation as

$$
\tan \left(k_{x} a\right)=\frac{\kappa}{k_{x}},
$$

as in the usual symmetric dielectric planar waveguide. Everything is identical to the standard theory, except the dispersion relation of the medium based on the expression $K_{\alpha}$ given by Eq. (15).
The guiding condition is, obviously, that both $k_{x}$ and $\kappa$ are real, i.e., $K_{1}>0$ and $K_{2}<0$. Taking for $t$ the retarded time, in the reference frame which moves at the velocity of linear low-frequency waves in the bulk medium from which the core is built, i.e., $V_{0}=V_{1}$, the velocity $v_{1}$ vanishes and the guiding condition reduces to

$$
A_{2} \omega^{3}-\omega v_{2}<k_{z}<A_{1} \omega^{3},
$$

and, in the special case $A_{1}=A_{2}$, it requires $v_{2}>0$. According to the above notations, the core and cladding indices are $n_{1}=$ $n_{0}$ and $n_{2}=n_{0}+\delta n_{2}$, where $v_{2}$ and $\delta n_{2}$ are related through Eq. (13) or Eq. (14). Let us call $\delta n=-\delta n_{2}>0$ the difference between the core and cladding refractive indices at the lowfrequency limit. The relative velocity $v_{2}$ is proportional to $\delta n$ according to (14), and the condition (17) reduces to the usual one, in which $\delta n$ must be a positive number.

\section{NONLINEAR WIDENING OF THE LINEAR GUIDED MODES}

The CGKP equation (4) is solved by means of the fourthorder Runge-Kutta exponential time differencing (RK4ETD) scheme [55]. It involves one integration with respect to $t$. The antiderivative is computed by means of a Fourier transform, which implies that the integration constant is fixed so that the mean value of the antiderivative is zero, but also that the linear term is replaced with zero, i.e., the mean value of the function $\partial_{x}^{2} u$ is set to zero. For low frequencies, the coefficients of the RK4ETD scheme are computed by means of series expansions to avoid catastrophic consequences of limited numerical accuracy. In order to reduce unphysical behavior due to the reinjection of dispersed waves, we use a quite long numerical box (100 times the dimensionless fundamental optical period $T$ ). However, for the computation of the antiderivative, we use no better accuracy in frequency than $v_{0} / 25$, where $v_{0}=1 / T$ is the fundamental frequency.

We consider input data in the form of a fundamental linear guided mode multiplied by a temporal Gaussian profile, as

$$
u(x, t, z=0)=u_{0}(x, t)=A \cos (\omega t) f(x) e^{-t^{2} / w^{2}},
$$

with

$$
f(x)=\left\{\begin{array}{lll}
\cos \left(k_{x} x\right), & \text { for } & |x| \leqslant a, \\
\cos \left(k_{x} a\right) e^{\kappa(a-|x|)}, & \text { for } & |x|>a .
\end{array}\right.
$$

Here $k_{x}$ and $\kappa$ are computed using Eq. (15), obviously. Hence $f$ is normalized so that $\max _{x}|f(x)|=1$ and $\max _{x, t}\left|u_{0}\right|=A$.

We use normalized coefficients $A_{1}=A_{2}=B_{1}=B_{2}=$ $W_{1}=W_{2}=1$, assuming that both the dispersion parameter and the nonlinear susceptibility have the same values in the waveguide core and in the cladding. We perform the computation in the reference frame, moving at the velocity of linear long wave in the waveguide core, i.e., $v_{1}=0$, and we consider several numerical values of the cladding velocity shift $v_{2}$, i.e., of the refractive index difference $\delta n$ between core and cladding. We check, by using $1 / w^{2}=0$ and very small values of $A$, that the model correctly accounts for the linear waveguiding, and, by setting $v_{2}$ to zero, we check that the diffraction effect is correctly described in both linear and nonlinear regimes in the absence of the waveguide. 


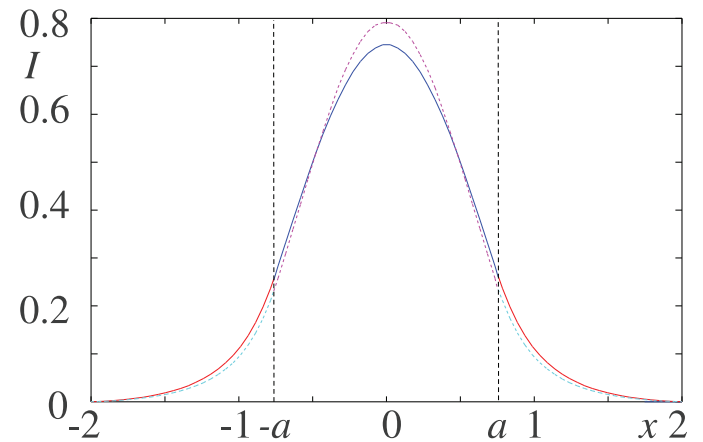

FIG. 1. (Color online) Guided wave profiles in the linear (dashed pink and cyan lines) and nonlinear (solid blue and red lines) regimes. Intensities are normalized so that the total power is 1 . Here $v_{2}=3$ and $w=4$.

We first consider $v_{2}=30$. We consider as a typical value of the dispersion parameter $2 n n^{\prime \prime}=0.015 \mathrm{fs}^{2}$; note that the values obtained in Ref. [30] in the BBO (beta barium borate) crystal at $\lambda=1 \mu \mathrm{m}$, using Sellmeier approximations of the dispersion relation given by industrials [56], were $2 n n^{\prime \prime}=-0.0111 \mathrm{fs}^{2}$ and $-0.0180 \mathrm{fs}^{2}$ for the ordinary and extraordinary indices, respectively. We also assume a reference time $\tau_{w}=0.8$ fs and a refractive index $n=1.6$; then $v_{2}=30$ corresponds to a large value of the index difference of about $\delta n=0.1$. The other parameters are $w=4, a=0.76, \omega=1$, and $A=1.2$, which would correspond to a pulse width (full width at half maximum, FWHM) of $3.77 \mathrm{fs}$, and a total core width of $5.3 \mu \mathrm{m}$. The reference lengths used in the normalization are, indeed, for the proposed parameters $l=3.5 \mu \mathrm{m}$ and $L=82 \mu \mathrm{m}$. We compute the confinement factor

$$
C F=\frac{\int_{-a}^{a} d x \int_{-\infty}^{+\infty} d t|u|^{2}}{\int_{-\infty}^{+\infty} d x \int_{-\infty}^{+\infty} d t|u|^{2}}
$$

(with the infinity symbol holding in practice for the boundary of the computation box), and we get $C F=98.7 \%$ in both linear and nonlinear regimes. We thus conclude that the confinement is very high. The mode profile is almost unchanged in the nonlinear regime.

Then we set $v_{2}=3$, i.e., we decrease $\delta n$ by one order of magnitude ( $\delta n=0.01$ with the above assumptions). The confinement factor decreases from $87.8 \%$ in the linear regime to $85.7 \%$ in the nonlinear one. The profile is slightly enlarged in the nonlinear regime; see Fig. 1. The intensities are computed by integration (summation) of $|u|^{2}$ over $t$, at the end of the computed evolution. Next we consider a much shorter pulse $(w=2)$, which requires a higher amplitude for soliton formation, $A=2.4$. It corresponds to a FWHM of 1.88 fs for the proposed parameters and, in this case, the confinement factor decreases in the linear regime $(80 \%)$ and even more in the nonlinear regime ( $70 \%$ only). The corresponding widening of the pulse profile is shown in Fig. 2.

If we decrease the width of the waveguide, the confinement factor still decreases. For $a=0.55$, that would correspond to a total width of $3.85 \mu \mathrm{m}$ and, as far as we can judge from our computations, guiding still occurs. However, the confinement factor goes down to $74 \%$ in the linear regime, and to only $60 \%$ in the nonlinear one. In our computations, the waves remain

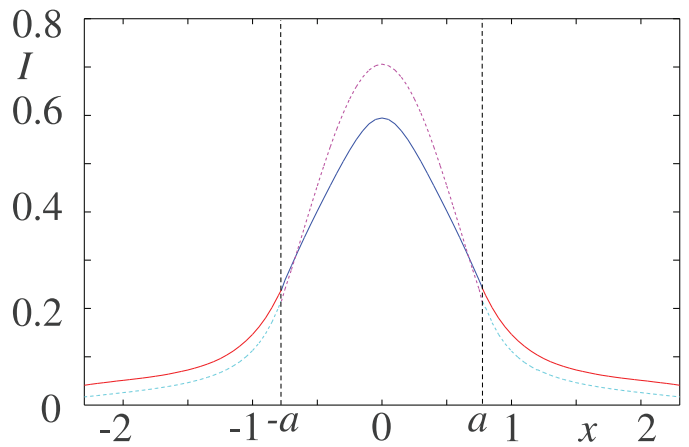

FIG. 2. (Color online) Same as Fig. 1, but for a shorter pulse. Here $v_{2}=3$ and $w=2$.

confined within the finite computation box due to energy conservation in the numerical model, even for a zero value of the index difference. For the present parameters, especially for our choice of guide width, the value of the confinement factor due to this numerical artifact is about $50 \%$, instead of 0 as it should be if the wave were allowed to diffract indefinitely as is physically the case. This is a limitation for the discrimination, in a numerical study, between low-confinement guided modes and the absence of guiding.

\section{THE STUDY OF THE NONLINEAR WAVEGUIDING PROCESS}

The fact that the mode profiles become wider and the confinement factor decreases in the nonlinear regime is easy to explain: it is well known that waveguiding is due to total internal reflection. The latter occurs when the wave propagates too fast in the cladding, so that it cannot be matched with the field oscillations in the core, whatever the propagation direction in the cladding is. In the few-cycle regime, not only does the linear phase velocity have to be taken into account, but so does the whole wave velocity, including its nonlinear component. Due to the sign of nonlinearity, the nonlinear velocity is positive and has a larger magnitude where the amplitude is higher, i.e., in the core. It creates a nonlinear variation of the refractive index, which may be comparable in magnitude to the linear part, as well as compensate it, reduce the efficiency of the guiding effect, and ultimately prevent it.

One might think that a change in the sign of the nonlinear coefficient would allow one to restore the waveguiding; however, we want to produce solitons and hence it would imply that one would also change the sign of dispersion. In this case, wave collapse occurs. The latter does not happen for picosecond solitons in fibers because the amplitude is much lower, and the transverse confinement which could arise due to self-focusing is not as small as the core width of a standard single-mode fiber.

One way to restore the waveguiding and improve the confinement in the nonlinear situation is to take advantage of the nonlinear velocity by using a higher nonlinear coefficient in the cladding than that in the core. Figure 3 shows the evolution of the guided mode profile against the nonlinear coefficient difference $B_{2}-B_{1}$. It is seen that, for a large value of this difference, the guided mode profile is the same as for the 


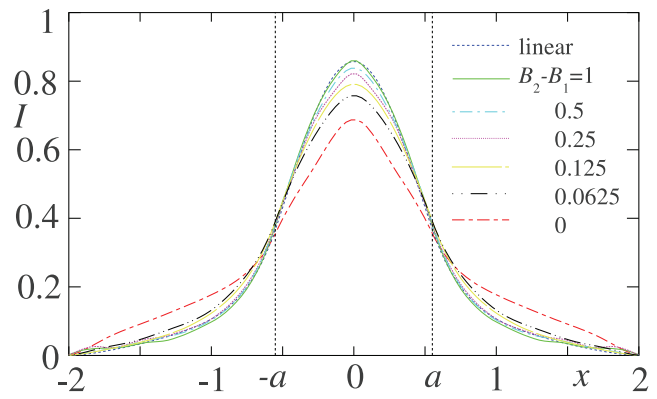

FIG. 3. (Color online) Guided profiles of the nonlinear waveguide for different values of the nonlinear coefficient difference. The linear guided profile is given for the sake of comparison. Intensities are normalized so that the total power is 1 .

linear guide, while the nonlinear confinement becomes less efficient for a smaller difference (see Table I).

However, not all of the energy of the input is guided. A part of it is dispersed and is not trapped in the temporal soliton. Since the guiding is mainly due to nonlinearity, and the radiated waves do not have sufficient peak power to induce the latter, they are not guided and they diffract. However, as mentioned above, they remain confined in the computation box instead of being totally spread out by diffraction. We thus have to remove them in an ad hoc way. To obtain the results above, we have suppressed the dispersed part (by setting $u$ to zero out of the main pulse) after a propagation distance of $z \simeq 50$ (it corresponds to $4.1 \mathrm{~mm}$ for the parameters above) and run again the computation with the "cleaned" pulse as initial data (note that we were not able to suppress totally the radiated waves). The numerical picture of the guided soliton obtained this way is displayed in Fig. 4. If we try to compute the confinement factor without removing the dispersed part of the input, we get a value close to the one due to the linear guide itself; it is, in fact, not significant, being a mix of guided modes and waves trapped in the computation box.

By using this guiding process, it is possible to reduce the refractive index difference $\delta n$ (or $v_{2}$ ). For $v_{2}=0.7$, which corresponds to $\delta n=0.0025$ with the above assumptions, and using the dimensionless guide width $a=0.775$ (i.e., a core width of $5.4 \mu \mathrm{m}$ ), we find that the confinement factor for the fundamental mode of the linear guide, for a plane wave, is $63.3 \%$. For a FCP with duration $w=2$ (the FWHM would be $1.9 \mathrm{fs}$ ), it decreases down to $55.8 \%$ in the linear regime. In the nonlinear regime, the remaining confinement we observe numerically is due in large part, and maybe in totality, to reflexions on the boundaries of the computation box. Thus we expect that, if it were possible to increase indefinitely the width of the computation box, it would go to zero; note that the reflexions on the boundary of the computation box are indeed clearly observed in our numerical resolutions. Let us consider now the nonlinear waveguide: the same amplitude

TABLE I. The confinement factor $C F$ of the nonlinear waveguide vs the nonlinear coefficient difference $\left(B_{2}-B_{1}\right)$.

\begin{tabular}{lccccccr}
\hline \hline$\left(B_{2}-B_{1}\right)$ & 1 & $1 / 2$ & $1 / 4$ & $1 / 8$ & $1 / 16$ & 0 & linear \\
\hline$C F(\%)$ & 74.4 & 71.9 & 73.3 & 70.7 & 68.3 & 60.2 & 74.0 \\
\hline \hline
\end{tabular}

$A=2.4$ which allows the formation of the soliton in both the $(1+1)$-dimensional reduction of the problem and in the guide with higher linear confinement is neither large enough to form a soliton, nor to produce nonlinear waveguiding. However, for a higher field intensity (we used $A=3$ ), the soliton forms and nonlinear waveguiding occurs. The confinement factor is rather low (33.7\%), but the guiding is effective. The corresponding numerical results are shown in Figs. 5-7.

\section{ANALYTIC THEORY FOR GUIDED FEW-CYCLE SOLITONS IN ULTRANARROW PLANAR WAVEGUIDES}

We consider that each medium in both the waveguide core and the cladding is described by a two-level Hamiltonian,

$$
H_{0, \alpha}=\hbar\left(\begin{array}{cc}
\omega_{\alpha, a} & 0 \\
0 & \omega_{\alpha, b}
\end{array}\right) .
$$

The electromagnetic field is coupled to the atoms by means of the dipolar momentum matrix

$$
\mu_{\alpha}=\hbar\left(\begin{array}{cc}
0 & \mu_{\alpha} \\
\mu_{\alpha}^{*} & 0
\end{array}\right),
$$

so that the evolution of the density matrix $\rho$ is given by

$$
i \hbar \partial_{\tau} \rho=\left[H_{0}-\mu E, \rho\right],
$$

in which $H_{0}$ and $\mu$ are $H_{0,1}$ and $\mu_{1}$ in the core and $H_{0,2}$ and $\mu_{2}$ in the cladding, respectively. The electric field $E$ (treated as a scalar) obeys the wave equation

$$
\left(\partial_{\xi}^{2}+\partial_{\zeta}^{2}\right) E=\frac{1}{c^{2}} \partial_{\tau}^{2}\left(E+\frac{P}{\varepsilon_{0}}\right),
$$

where the polarization density $P$ satisfies

$$
P=N_{\alpha} \operatorname{Tr}\left(\rho \mu_{\alpha}\right),
$$

with $N_{\alpha}$ being the number of atoms per volume unit.

According to the reductive perturbation method [57], we introduce slow variables $t$ and $z$,

$$
t=\varepsilon\left(\tau-\frac{\zeta}{V}\right), z=\varepsilon^{3} \zeta,
$$

in both the waveguide core $(|\xi| \leqslant a)$ and in the cladding $(|\xi| \geqslant$ $a$ ), and the slow variable $x$,

$$
x=\varepsilon \xi,
$$

in the cladding only, with the waveguide core being assumed to be too narrow to allow variations in the $\xi$ direction. The electric field is expanded in a power series of $\varepsilon$ as $E=\varepsilon E_{1}+$ $\varepsilon^{2} E_{2}+\cdots$, and so on. We denote the components of the matrix $\rho$ as

$$
\rho=\left(\begin{array}{cc}
\rho_{a} & \rho_{t} \\
\rho_{t}^{*} & \rho_{b}
\end{array}\right)
$$

and so on.

Equation (22) at order $\varepsilon^{0}$ merely shows that the coherence term $\rho_{0, t}$ must be zero. The diagonal terms $\rho_{\alpha, a, 0}, \rho_{\alpha, b, 0}$ are constant in both media and equal to the values of the populations at thermal equilibrium.

Equation (22) at order $\varepsilon^{1}$ gives, as in Ref. [58],

$$
\rho_{t, 1}=\frac{-E_{1} \mu_{\alpha} w_{\alpha, 0}}{\hbar \Omega_{\alpha}},
$$



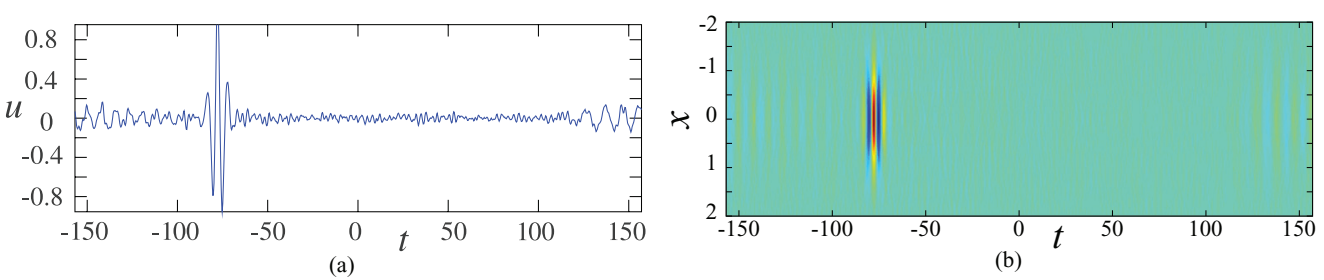

FIG. 4. (Color online) (a) The temporal profile and (b) the spatiotemporal profile of the guided FCP soliton in the case of a nonlinear waveguide with $B_{2}-B_{1}=1$.

where $\Omega_{\alpha}=\omega_{\alpha, b}-\omega_{\alpha, a}>0$, and $w_{\alpha, 0}=\rho_{\alpha, b, 0}-\rho_{\alpha, a, 0}$. This allows one to compute $P_{1}$ as

$$
P_{1}=\varepsilon_{0}\left(n_{\alpha}^{2}-1\right) E_{1}
$$

where the optical index $n_{\alpha}$ is given by

$$
n_{\alpha}^{2}=1-\frac{2 N_{\alpha} w_{\alpha, 0}\left|\mu_{\alpha}\right|^{2}}{\hbar \Omega_{\alpha}} .
$$

The obtained expression of $P_{1}$ is inserted into the wave equation (23) at leading order $\varepsilon^{3}$. In the core $(\alpha=1)$, we merely get the velocity as $V=c / n_{1}$. In the cladding $(\alpha=2)$, the situation differs because $V$ is already fixed in the core, and a term $\partial_{x}^{2} E_{1}$ appears. Defining

$$
K=\frac{n_{1}^{2}-n_{2}^{2}}{c^{2}}
$$

the wave equation reduces to

$$
\partial_{x}^{2} E_{1}+K \partial_{t}^{2} E_{1}=0 .
$$

Equation (22) at order $\varepsilon^{2}$ gives $\rho_{a, 1}=\rho_{b, 1}=0$ and

$$
\rho_{t, 2}=\frac{-\mu_{\alpha} w_{\alpha, 0}}{\hbar \Omega_{\alpha}} E_{2}+\frac{i \mu_{\alpha} w_{\alpha, 0}}{\hbar \Omega_{\alpha}^{2}} \partial_{t} E_{2}
$$

from which we deduce

$$
P_{2}=\varepsilon_{0}\left(n_{\alpha}^{2}-1\right) E_{2} .
$$

We see that Eq. (23) at order $\varepsilon^{4}$ is automatically satisfied in the waveguide core, and it reduces to the same equation as (31) to be satisfied by $E_{2}$ in the cladding.

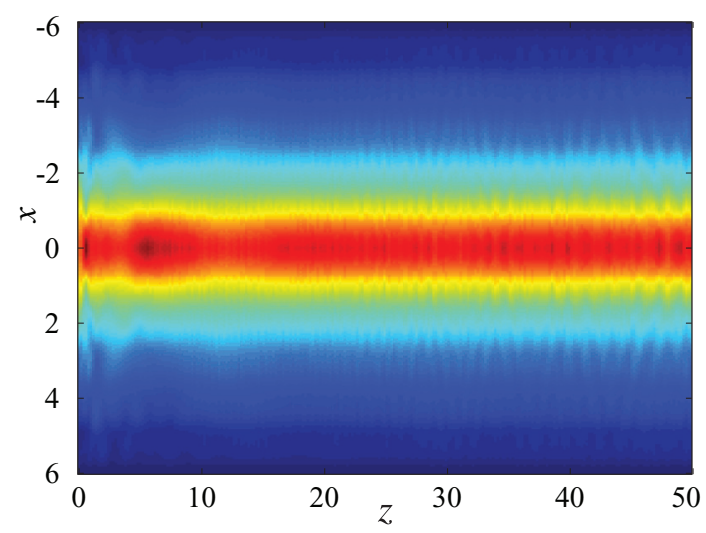

FIG. 5. (Color online) The evolution of the spatial distribution of optical average intensity in the propagation along $z$ axis in the case of the nonlinear waveguide with $v_{2}=0.7$ and $B_{2}-B_{1}=1 / 4$.
Then, Eq. (22) at order $\varepsilon^{3}$ provides the following expressions, in the same way as in the case of the bulk medium [58]:

$$
\begin{gathered}
\rho_{a, 2}=-\rho_{b, 2}=\frac{\left|\mu_{\alpha}\right|^{2} w_{\alpha, 0}}{\hbar^{2} \Omega_{\alpha}^{2}} E_{1}^{2}, \\
\rho_{t, 2}=\frac{-\mu_{\alpha} w_{\alpha, 0}}{\hbar \Omega_{\alpha}} E_{3}+\frac{i \mu_{\alpha} w_{\alpha, 0}}{\hbar \Omega_{\alpha}^{2}} \partial_{t} E_{2}+\frac{\mu_{\alpha} w_{\alpha, 0}}{\hbar \Omega_{\alpha}^{3}} \partial_{t}^{2} E_{1} \\
+\frac{2 \mu_{\alpha}\left|\mu_{\alpha}\right|^{2} w_{\alpha, 0}}{\hbar^{3} \Omega_{\alpha}^{3}} E_{1}^{3},
\end{gathered}
$$

from which we deduce

$$
\begin{aligned}
P_{3}= & \varepsilon_{0}\left(n_{\alpha}^{2}-1\right) E_{3}+\frac{2 N_{\alpha}\left|\mu_{\alpha}\right|^{2} w_{\alpha, 0}}{\hbar \Omega_{\alpha}^{3}} \partial_{t}^{2} E_{1} \\
& +\frac{4 N_{\alpha}\left|\mu_{\alpha}\right|^{4} w_{\alpha, 0}}{\hbar^{3} \Omega_{\alpha}^{3}} E_{1}^{3} .
\end{aligned}
$$

Finally, Eq. (23) at order $\varepsilon^{5}$ reduces, in the waveguide core, to the modified Korteweg-de Vries (mKdV) equation

$$
\partial_{z} E_{1}+\beta \partial_{t}^{3} E_{1}+\gamma \partial_{t} E_{1}^{3}=0,
$$

with

$$
\begin{gathered}
\beta=\frac{V N_{1}\left|\mu_{1}\right|^{2} w_{1,0}}{\varepsilon_{0} c^{2} \hbar \Omega_{1}^{3}}, \\
\gamma=\frac{2 V N_{1}\left|\mu_{1}\right|^{4} w_{1,0}}{\varepsilon_{0} c^{2} \hbar^{3} \Omega_{1}^{3}} .
\end{gathered}
$$

The mKdV equation (37) to be satisfied at $x=0$, together with Eq. (31) to be satisfied for nonzero $x$, yield the sought system

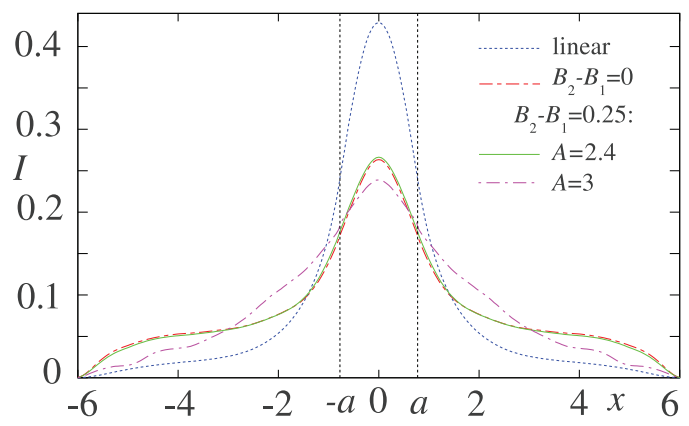

FIG. 6. (Color online) The transverse profiles for a guide with low refractive index difference $\left(v_{2}=0.7\right)$ : linear regime, nonlinear regime without nonlinear waveguiding, nonlinear waveguide with insufficient power to form a guided FCP soliton $(A=2.4)$, and nonlinearly guided FCP soliton $(A=3)$. 

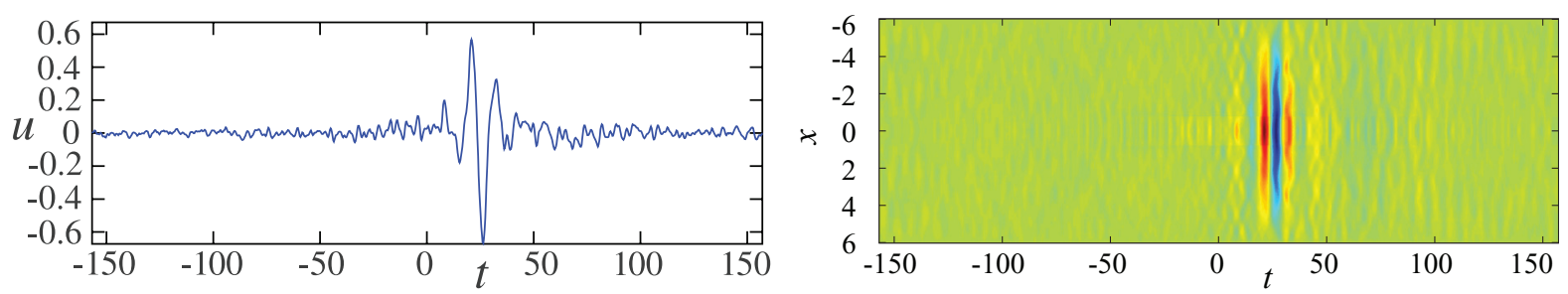

FIG. 7. (Color online) (a) The temporal profile and (b) the spatiotemporal profile of the guided FCP soliton in the case of the nonlinear waveguide with $v_{2}=0.7$ and $B_{2}-B_{1}=1 / 4$.

of equations for the field $E_{1}$. We fix a reference slow time $t_{w}$; then the change of variables,

$$
T=\frac{t}{t_{w}}, \quad Z=\frac{\beta}{t_{w}^{3}} z, \quad u=t_{w} \sqrt{\frac{\gamma}{2 \beta}} E_{1}, \quad X=\frac{\sqrt{K}}{t_{w}} x,
$$

reduces Eqs. (37) and (31) to the dimensionless system of equations,

$$
\begin{gathered}
\partial_{Z} u+\partial_{T}^{3} u+2 \partial_{T} u^{3}=0, \\
\partial_{X}^{2} u+\partial_{T}^{2} u=0 .
\end{gathered}
$$

It is worthwhile to note that Eq. (42) shows that $u$ must be a harmonic function in each half plane $\operatorname{Re}(Y)>0$ and $\operatorname{Re}(Y)<$ 0 , where the complex variable $Y$ is $Y=T+i X$.

Let us consider the linear regime, i.e., let us replace $\gamma$ with zero, or remove the nonlinear term in Eq. (41). A solution of (41) is $u_{0}=C e^{i\left(\omega T-\omega^{3} Z\right)}$, and then it is easily seen that the corresponding solution of Eq. (42) is

$$
u_{0}=C \exp \left[i\left(\omega T-\omega^{3} Z\right)-\omega|X|\right],
$$

assuming $\omega>0$.

In the general case, Eq. (42) can be solved as follows: we consider the Fourier expansion of $u_{0}(T)=u(T, X=0)$ as

$$
u_{0}(T)=\int_{-\infty}^{+\infty} \hat{u}_{0}(\omega) e^{i \omega T} d \omega
$$

Let $U_{0}$ be the part of $u_{0}$ corresponding to positive frequencies, i.e.,

$$
U_{0}(T)=\int_{0}^{+\infty} \hat{u}_{0}(\omega) e^{i \omega T} d \omega
$$

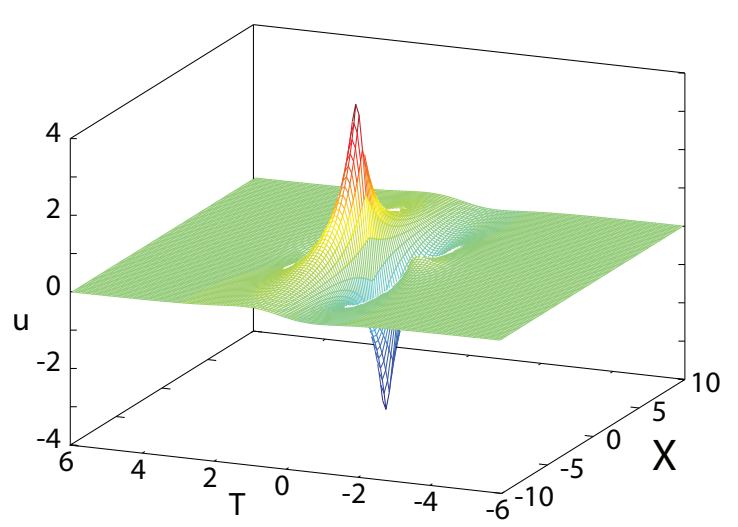

FIG. 8. (Color online) The guided FCP as described by the approximate analytical approach.
Since $u_{0}$ is real, we easily see that $u_{0}=2 \operatorname{Re}\left(U_{0}\right)$.

The solution of Eq. (42) pertaining to $U_{0}$ is

$$
U(T, X)=\int_{0}^{+\infty} \hat{u}_{0}(\omega) e^{i \omega T-\omega|X|} d \omega,
$$

i.e., $U(T, X)=U_{0}(T+i X)$ if $X \geqslant 0$, and $U(T, X)=$ $U_{0}(T-i X)$ if $X \leqslant 0$. Then,

$$
u(T, X, Z)=2 \operatorname{Re}\left[U_{0}(T+i|X|, Z)\right]
$$

is the complete solution. The computation of Eq. (47) can be easily implemented numerically.

The two-soliton solution of Eq. (41) is [59]

$$
u=\frac{e^{\eta_{1}}+e^{\eta_{2}}+\left(\frac{p_{1}-p_{2}}{p_{1}+p_{2}}\right)^{2}\left(\frac{e^{\eta_{1}}}{4 p_{1}^{2}}+\frac{e^{\eta_{2}}}{4 p_{2}^{2}}\right) e^{\eta_{1}+\eta_{2}}}{1+\frac{e^{2 \eta_{1}}}{4 p_{1}^{2}}+\frac{2}{\left(p_{1}+p_{2}\right)^{2}} e^{\eta_{1}+\eta_{2}}+\frac{e^{2 \eta_{2}}}{4 p_{2}^{2}}+\left(\frac{p_{1}-p_{2}}{p_{1}+p_{2}}\right)^{4} \frac{e^{2 \eta_{1}+2 \eta_{2}}}{16 p_{1}^{2} p_{2}^{2}}},
$$

with $\eta_{j}=p_{j} T-p_{j}^{3} Z-\gamma_{j}$, for $j=1$ and 2 . If we choose the arbitrary parameters $p_{1}, p_{2}, \gamma_{1}$, and $\gamma_{2}$ so that $p_{2}=p_{1}^{*}$ and $\gamma_{2}=\gamma_{1}^{*}$, expression (48) is the breather solution of the $\mathrm{mKdV}$ equation. An example of the computation with $p_{1}=3+2 i$, $\gamma_{1}=0$, and $Z=0.2$ is shown in Fig. 8 .

The mode width is typically $1 / \omega$ in the dimensionless variable $X$, which reads, if we come back to the laboratory frame variable, as

$$
l_{m}=\frac{\lambda}{2 \pi \sqrt{n_{1}^{2}-n_{2}^{2}}},
$$

where the quantity $\lambda=2 \pi t_{w} c /(\varepsilon \omega)$ has been identified as the central wavelength of the pulse. If we compare Eq. (49) to the usual expression of the normalized frequency, $V=$ $2 a(2 \pi / \lambda) \sqrt{n_{1}^{2}-n_{2}^{2}}$, we find $l_{m}=2 a / V$, which is the typical order of magnitude given by the exact linear theory. Thus this approximate analytical approach allows one to describe guided FCP soliton propagation, but is not able to account for the transverse widening of the mode which occurs in the nonlinear regime.

\section{CONCLUSIONS}

In this work, we considered both linear and nonlinear waveguiding of few-cycle spatiotemporal wave packets in a planar geometry. In order to avoid the wave collapse, we restricted ourselves to the case of self-defocusing Kerr nonlinearity of the medium and to normal group-velocity dispersion. The evolution of the electric field in the cubic (Kerr) nonlinear 
medium was described by the cubic generalized KadomtsevPetviashvili equation, which was solved by the fourth-order Runge-Kutta exponential time differencing method. We have found that for the linear waveguide in the nonlinear regime, the mode profiles are much wider and the confinement factor decreases in comparison to the situation of the linear regime. For narrow waveguides in the subcycle regime, it may happen that the wave is not guided at all. However, in the study of propagation of few-cycle spatiotemporal pulses, not only does the linear phase velocity have to be taken into account, but also does the nonlinear component of the whole wave velocity, and therefore we have shown that it was possible to recover the guiding and to improve the confinement factor in the nonlinear regime by using a higher nonlinear coefficient in the cladding than that in the waveguide core, i.e., by realizing a truly nonlinear waveguide. An analytic approach for guided few-cycle solitons in ultranarrow planar waveguides was introduced too. It has the advantage of allowing a simple description of the guided few-cycle solitons, but does not account for the mode widening and nonlinear waveguiding discussed above. The results obtained in this work can be relatively easily extended to other relevant waveguiding geometries, e.g., to both rectangular and cylindrical channel optical waveguides.

\section{ACKNOWLEDGMENTS}

The work of D.M. was supported in part by the Romanian Ministry of Education and Research (Project No. PN-II-IDPCE-2011-3-0083).
[1] G. I. Stegeman, D. N. Christodoulides, and M. Segev, IEEE J. Sel. Top. Quantum Electron. 6, 1419 (2000).

[2] Y. S. Kivshar and G. P. Agrawal, Optical Solitons: From Fibers to Photonic Crystals (Academic, San Diego, 2003).

[3] H. G. Purwins, H. U. Bodeker, and Sh. Amiranashvili, Adv. Phys. 59, 485 (2010).

[4] Y. V. Kartashov, B. A. Malomed, and L. Torner, Rev. Mod. Phys. 83, 247 (2011).

[5] Z. Chen, M. Segev, and D. N. Christodoulides, Rep. Prog. Phys. 75, 086401 (2012).

[6] A. Kujawski, Z. Phys. 66, 271 (1987).

[7] A. V. Andreev, Zh. Éksp. Teor. Fiz. 108, 796 (1995) [JETP 81, 434 (1995)].

[8] A. Yu. Parkhomenko and S. V. Sazonov, Zh. Éksp. Teor. Fiz. 114, 1595 (1998) [JETP 87, 864 (1998)].

[9] N. N. Akhmediev, I. V. Mel'nikov, and A. V. Nazarkin, Sov. Phys. Lebedev Inst. Rep. 2, 66 (1989) [Kratk. Soobshch. Fiz. FIAN 2, 49 (1989)].

[10] E. M. Belenov and A. V. Nazarkin, Pis'ma Zh. Éksp. Teor. Fiz. 51, 252 (1990) [JETP Lett. 51, 288 (1990)].

[11] I. V. Melnikov, D. Mihalache, F. Moldoveanu, and N.-C. Panoiu, Phys. Rev. A 56, 1569 (1997).

[12] I. V. Mel'nikov, D. Mihalache, and N.-C. Panoiu, Opt. Commun. 181, 345 (2000).

[13] H. Leblond and D. Mihalache, Phys. Rep. 523, 61 (2013).

[14] N. N. Rosanov, V. V. Kozlov, and S. Wabnitz, Phys. Rev. A 81, 043815 (2010).

[15] H. Leblond and D. Mihalache, J. Phys. A: Math. Theor. 43, 375205 (2010).

[16] V. V. Kozlov, N. N. Rosanov, and S. Wabnitz, Phys. Rev. A 84, 053810 (2011).

[17] Sh. Amiranashvili, U. Bandelow, and N. Akhmediev, Phys. Rev. A 84, 043834 (2011).

[18] H. Leblond and D. Mihalache, Rom. Rep. Phys. 63, 1254 (2011).

[19] A. A. Drozdov, S. A. Kozlov, A. A. Sukhorukov, and Y. S. Kivshar, Phys. Rev. A 86, 053822 (2012).

[20] Y. Shen, N. Whitaker, P. G. Kevrekidis, N. L. Tsitsas, and D. J. Frantzeskakis, Phys. Rev. A 86, 023841 (2012).

[21] M. Kolesik, P. Jakobsen, and J. V. Moloney, Phys. Rev. A 86, 035801 (2012).
[22] P. Whalen, J. V. Moloney, A. C. Newell, K. Newell, and M. Kolesik, Phys. Rev. A 86, 033806 (2012).

[23] D. V. Novitsky, Phys. Rev. A 86, 063835 (2012).

[24] A. Pusch, I. V. Shadrivov, O. Hess, and Y. S. Kivshar, Opt. Express 21, 1121 (2013)

[25] V. V. Kozlov and N. N. Rosanov, Phys. Rev. A 87, 043836 (2013).

[26] F. Kimiaee Asadi, B. Shokri, and H. Leblond, Opt. Commun. 294, 283 (2013).

[27] Y. Xiao, D. N. Maywar, and G. P. Agrawal, Opt. Lett. 38, 724 (2013).

[28] Sh. Amiranashvili, U. Bandelow, and N. Akhmediev, Phys. Rev. A 87, 013805 (2013).

[29] H. Triki, H. Leblond, and D. Mihalache, Opt. Commun. 285, 3179 (2012).

[30] H. Leblond, H. Triki, and D. Mihalache, Phys. Rev. A 85, 053826 (2012).

[31] H. Leblond, H. Triki, and D. Mihalache, Phys. Rev. A 86, 063825 (2012).

[32] X. Liu, L. J. Qian, and F. W. Wise, Phys. Rev. Lett. 82, 4631 (1999).

[33] A. Chong, W. H. Renninger, D. N. Christodoulides, and F. W. Wise, Nat. Photon. 4, 103 (2010).

[34] D. Abdollahpour, S. Suntsov, D. G. Papazoglou, and S. Tzortzakis, Phys. Rev. Lett. 105, 253901 (2010).

[35] S. Minardi, F. Eilenberger, Y. V. Kartashov, A. Szameit, U. Röpke, J. Kobelke, K. Schuster, H. Bartelt, S. Nolte, L. Torner, F. Lederer, A. Tünnermann, and T. Pertsch, Phys. Rev. Lett. 105, 263901 (2010).

[36] F. Eilenberger, S. Minardi, A. Szameit, U. Röpke, J. Kobelke, K. Schuster, H. Bartelt, S. Nolte, L. Torner, F. Lederer, A. Tünnermann, and T. Pertsch, Phys. Rev. A 84, 013836 (2011).

[37] F. Eilenberger, S. Minardi, A. Szameit, U. Röpke, J. Kobelke, K. Schuster, H. Bartelt, S. Nolte, A. Tünnermann, and T. Pertsch, Opt. Express 19, 23171 (2011).

[38] W. H. Renninger and F. W. Wise, Nat. Commun. 4, 1719 (2013).

[39] Y. Silberberg, Opt. Lett. 15, 1282 (1990).

[40] A. B. Blagoeva, S. G. Dinev, A. A. Dreischuh, and A. Naidenov, IEEE J. Quantum Electron. 27, 2060 (1991). 
[41] D. E. Edmundson and R. H. Enns, Opt. Lett. 17, 586 (1992).

[42] N. Akhmediev and J. M. Soto-Crespo, Phys. Rev. A 47, 1358 (1993).

[43] R. McLeod, K. Wagner, and S. Blair, Phys. Rev. A 52, 3254 (1995).

[44] B. A. Malomed, D. Mihalache, F. Wise, and L. Torner, J. Opt. B: Quantum Semiclassical Opt. 7, R53 (2005).

[45] D. Mihalache, Rom. J. Phys. 57, 352 (2012); Rom. Rep. Phys. 63, 9 (2011); Proc. Romanian Acad. A 11, 142 (2010).

[46] H. Leblond, D. Kremer, and D. Mihalache, Phys. Rev. A 81, 033824 (2010).

[47] H. Leblond and D. Mihalache, Phys. Rev. A 81, 063815 (2010).

[48] H. Leblond, D. Kremer, and D. Mihalache, Phys. Rev. A 80, 053812 (2009).

[49] E. V. Kazantseva, A. I. Maimistov, and B. A. Malomed, Opt. Commun. 188, 195 (2001).

[50] H. Leblond and D. Mihalache, Phys. Rev. A 86, 043832 (2012).
[51] O. Fedotova, A. Husakou, and J. Herrmann, Opt. Express 14, 1512 (2006).

[52] S.-S. Yu, C.-H. Chien, Y. Lai, and J. Wang, Opt. Commun. 119, 167 (1995).

[53] S. Raghavan and G. P. Agrawal, Opt. Commun. 180, 377 (2000).

[54] S. A. Kozlov and S. V. Sazonov, Zh. Éksp. Teor. Fiz. 111, 404 (1997) [JETP 84, 221 (1997)].

[55] S. M. Cox and P. C. Matthews, J. Comput. Phys. 176, 430 (2002).

[56] http://www.redoptronics.com/BBO-crystal.html.

[57] H. Leblond, J. Phys. B: At. Mol. Opt. Phys. 41, 043001 (2008).

[58] H. Leblond and F. Sanchez, Phys. Rev. A 67, 013804 (2003).

[59] R. Hirota, Bäcklund Transformations, The Inverse Scattering Method, Solitons, and Their Applications (Workshop Contact Transformation, Vanderbilt University, Nashville, TN, 1974), Lecture Notes in Math Vol. 515 (Springer, Berlin, 1976), pp. $40-68$. 